\title{
A New Solution of Non-integrated Correlative Light and Electron Microscopy Based on High-vacuum Optical Platform
}

\author{
Shuoguo $\mathrm{Li}^{1}$, Gang $\mathrm{Ji}^{1}$, Xiaojun Huang ${ }^{1}$, Lei Sun ${ }^{1}$, Jianguo Zhang ${ }^{1}$, Wei Xu ${ }^{1}$, Fei Sun ${ }^{1}$. \\ 1. Center for Biological Imaging, Core Facilities for Protein Science, Institute of Biophysics, CAS, \\ Beijing, China.
}

\begin{abstract}
Correlative light and electron microscopy (CLEM) offers a means of guiding the search for the unique or rare events by fluorescence microscopy (FM) and allows electron microscopy (EM) to zoom in on them for subsequent EM examination in three-dimensions (3D) and with nanometer-scale resolution. FM visualizes the localization of specific antigens by using fluorescent tags or proteins in a large fieldof-view to study their cellular function, whereas EM provides the high level of resolution for complex structures. And cryo CLEM combines the advantages of maintaining structural preservation in a nearnative state throughout the entire imaging process and by avoiding potentially harmful pre-treatments, such as chemical fixation, dehydration and staining with heavy metals. Besides for frozen-hydrated biological samples, CLEM combines the advantages of a close-to-life preservation of biological materials by keeping them embedded in vitreous ice throughout the entire imaging process and the frozen-hydrated condition is very suitable to maintain fluorescent signals. In recent years, many new instruments and software which intended to optimize the workflow and to obtain better experimental results of CLEM have been presented or even commoditized. While, the specimen damage during transfer from FM to EM and the resolution of CLEM were still need to be improved.
\end{abstract}

Here we set up a High-vacuum Optical Platform to develop CLEM imaging technology (HOPE), which was designed to realize high-vacuum optical ( fluorescent) imaging for cryo-sample on EM cryo-holder (e.g. Gatan 626). A non-integrated high-vacuum cryo-optical stage, which adapted to the EM cryo holder, was fixed on epi-fluorescence microscope (or super-resolution microscope) to obtain fluorescent images. And then the EM cryo holder would be transferred to EM for collection of EM data. This protocol was aimed to minimize the specimen damage during transfer from FM to EM and it was versatile to expend to different types of light microscopy or electron microscopy. Our HOPE had already passed correlative imaging test, and the results showed that it was convenient and effective.

\section{References:}

[1] Anna Sartori, Rudolf Gatz, Florian Beck, Alexander Rigort, Wolfgang Baumeister, Juergen M. Plitzko. Correlative microscopy: Bridging the gap between fluorescence light microscopy and cryoelectron tomography, Journal of Structural Biology 160 (2007), p. 135-145

[2] Peijun Zhang. Correlative cryo-electron tomography and optical microscopy of cells, Current Opinion in Structural Biology 23(2013), p. 763-770

[3] Kirsi Hellström, Helena Vihinen, Katri Kallio, Eija Jokitalo, Tero Ahola. Correlative light and electron microscopy enables viral replication studies at the ultrastructural level, Methods 90(2015), p. 49-56 


\section{Experimental and Results}
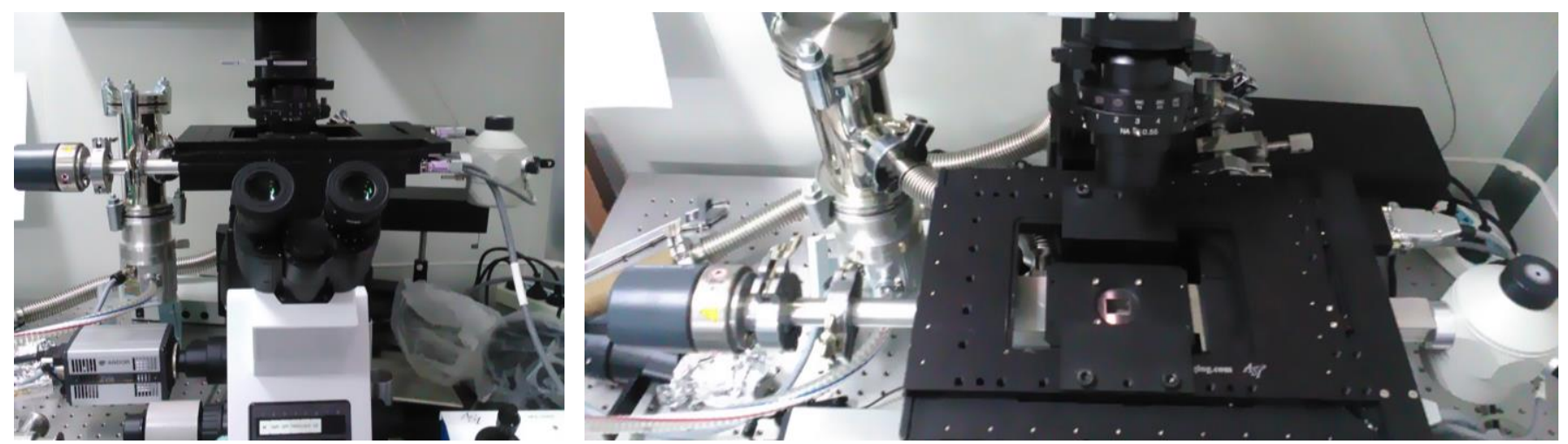

Figure 1. High-vacuum Optical Platform on epi-fluorescence microscope.
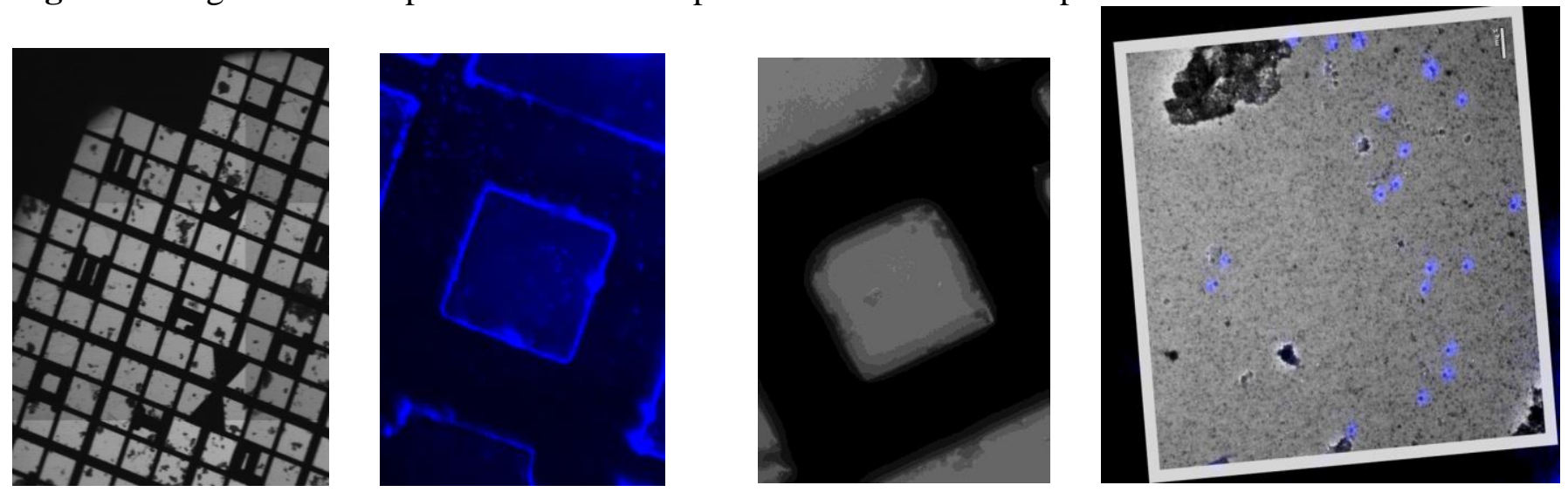

Figure 2. Blue fluorescent beads were used for positioning for FM and transmission EM imaging.

a. Low magnification (10X) imaging at Cryo-Stage of fluorescent beads sample in light microscope. $b$. Fluorescent imaging (40X long work distance objective lense ) of ROI in (a). C. TEM imaging (78X) of ROI in (a). d. Correlative Light and Electron Microscopy (CLEM) imaging of (b) and TEM imaging (6000X) of ROI in (c).

Sample preparation:

we used blue fluorescent beads (FluoSpheres ${ }^{\circledR}$ Carboxylate-Modified Microspheres, $0.2 \mu \mathrm{m}$, bluefluorescent (365/445), 2\% solids) for positioning in FM and TEM imaging.

Image Details:

Instrument used: Olympus IX73 Microscope (with Hi-Vac Cryo stage) \& FEI Tecnai 20 TEM (Scale bars: $2 \mu \mathrm{m})$. 